\title{
A BRIEF HISTORY OF THE POSITIVITY CONJECTURE IN TENSOR CATEGORY THEORY
}

\author{
GEOFFREY MASON \\ Department of Mathematics, UC Santa Cruz, CA 95064, USA. \\ E-mail: gem@ucsc.edu \\ |||||| \\ Abstract \\ We show the existence of a finite group $G$ having an irreducible character $\chi$ with \\ Frobenius-Schur indicator $\nu_{2}(\chi)=+1$ such that $\chi^{2}$ has an irreducible constituent $\varphi$ with \\ $\nu_{2}(\varphi)=-1$. This provides counterexamples to the positivity conjecture in rational CFT \\ and a conjecture of Zhenghan Wang about pivotal fusion categories.
}

\section{Introduction}

There are many positivity conjectures in mathematics. The one that concerns us here appeared at the turn of the century in a paper of Peter Bantay ([1], eqn(2)). It states that

$$
N_{p q}^{r} \nu_{p} \nu_{q} \nu_{r} \geq 0
$$

The context is nominally that of rational CFT: $p, q, r$ are simple objects with Frobenius-Schur indicators $\nu_{p}, \nu_{q}, \nu_{r}$ respectively, and $N_{p q}^{r}$ is the corresponding fusion rule. The conjecture is meaningful in tensor categories equipped with appropriate additional structures, and we shall view it in that context.

Soon after his paper appeared, Bantay brought the positivity conjecture to the author's attention. Framing it in a group-theoretic context, Bantay asked if one can find a finite group $G$ and a pair of irreducible characters $\alpha, \beta$ of $G$, each with Frobenius-Schur indicator +1 , such that $\alpha \beta$ has an

Received: 18 Jun 2017.

AMS Subject Classification: 18D10, 20G05.

Key words and phrases: Fusion category, positivity conjecture, Frobenius-Schur indicator.

The author thanks the Simon Foundation, grant \#427007, for its support. 
irreducible constituent with indicator -1 ? I was able to supply such a $G$, thereby falsifying (11). The construction was never published, but experts became aware of its existence.

In the meantime several variants of the positivity conjecture emerged. Already in 2001 Bantay had communicated a modified positivity conjecture to me, which he ascribed to 'string theorists'. This conjecture proposed that (11) holds whenever $N_{p q}^{r}$ is odd. This form of the conjecture is true and, as Richard Ng has pointed out, may be deduced from the final Theorem in a 2002 paper by Jürgen Fuchs, Ingo Runkel and Christoph Schweigert [4], who work in the context of a semisimple, braided, sovereign tensor category.

More recently, Zhenghan Wang conjectured a version in his 2006 book on topological quantum computation ([5], Conjecture 4.26) which may be stated as follows (we are in a pivotal fusion category):

$$
N_{p, p^{\vee}}^{r}>0 \Rightarrow \nu_{r}=1 .
$$

It transpires that the same counterexample to the positivity conjecture also serves as a counterexample to Wang's conjecture, and the purpose of this Note is to belatedly make this example public. We will show the following:

There is a pair $(G, \chi)$ consisting of a finite group $G$ (of order $2^{7}$ ) and an irreducible character $\chi$ of $G$ such that $\chi$ has indicator $\nu_{2}(\chi)=+1$ and $\chi^{2}$ has an irreducible constituent $\varphi$ with indicator $\nu_{2}(\varphi)=-1$.

We are indebted to Eric Rowell for bringing Wang's conjecture to our attention and Richard Ng for helpful correspondence.

\section{Construction}

We use standard facts from representation theory, but include some details for the sake of completeness.

Notation: if $G$ is a group and $a, b \in G$ then $[a, b]:=a^{-1} b^{-1} a b$. $\hat{G}:=\operatorname{Hom}\left(G, \mathbf{C}^{\times}\right)$is the group of characters of $G$; If $A \subseteq G$ is a subset of $G$, the centralizer of $A$ is $C_{G}(A):=\{g \in G \mid g a=a g(a \in A)\}$. In particular, the center of $G$ is $Z(G):=C_{G}(G)$. $Q_{8}$ : quaternion group of order 8 . 
$G L_{n}(2)$ : group of all $n \times n$ invertible matrices with entries in the field of 2 elements.

$S_{n}$ : symmetric group of degree $n ; A_{n}$ :alternating group of degree $n$.

1) Take $G$ to be any 2-group with a normal subgroup $H \unlhd G$ satisfying $G / H \cong Q_{8}, H \cong \mathbf{Z}_{2}^{4}$ and $C_{G}(H)=H$. For example, we may take $G=H \rtimes Q$ where $Q \cong Q_{8}$ acts faithfully (by conjugation) on $H$. (The existence of such a $G$ is shown below.)

2) Let $\lambda \in \hat{H}$ be such that for each $\operatorname{coset} x H \neq H$ in $G$ (alternatively, in the semi-direct product case, for $1 \neq x \in Q$ ) ker $\lambda$ does not contain $[H, x]$. (The existence of $\lambda$ is shown below. $[H, x]$ is defined below.)

Claim 1. The induced character $\chi:=\lambda^{G}:=\operatorname{Ind} d_{H}^{G} \lambda$ is irreducible.

Proof. One knows ([3], Corollary 10.20) that $\chi$ is irreducible, if, and only if, $\lambda \neq^{x} \lambda$ for all $x \in G \backslash H$. Here, ${ }^{x} \lambda$ is defined by

$$
{ }^{x} \lambda(h):=\lambda\left(x^{-1} h x\right) \quad(h \in H) .
$$

Now we have

$$
\begin{aligned}
& \lambda={ }^{x} \lambda \Leftrightarrow \lambda(h)={ }^{x} \lambda(h) \quad(h \in H) \\
& \Leftrightarrow \lambda(h)=\lambda\left(x^{-1} h x\right) \Leftrightarrow \lambda\left(h^{-1} x^{-1} h x\right)=1 \\
& \Leftrightarrow[H, x] \subseteq \text { ker } \lambda,
\end{aligned}
$$

where $[H, x]:=\langle[h, x] \mid h \in H\rangle$.

By 2) this never happens. So indeed $\chi$ is irreducible.

Claim 2. $\chi^{2}$ has an irreducible constituent $\varphi$ with $\nu_{2}(\varphi)=-1$.

Proof. Mackey has made a general study of the decomposition of tensor squares of induced characters such as $\chi$ ([3], Theorem 12.17). In particular, it is known (loc. cit.) that there is a constituent equal to $\left(\lambda^{2}\right)^{G}$. Now $\lambda$ is a character of $H$, which is a group of exponent 2. Therefore, $\lambda^{2}=1_{H}$. It follows that $\left(\lambda^{2}\right)^{G}=\left(1_{H}\right)^{G}=\rho_{G / H}=\rho_{Q}$ is the regular representation of $G / H$. This has every irreducible character of $Q_{8}$ as a constituent, and in particular it has a constituent with FS indicator equal to -1, namely the 2-dimensional irreducible $\varphi$ for $Q_{8}$ (lifted to $G$ ). 
Claim 3. $G$ exists.

Regarding $H$ as the natural module for $G L_{4}(2)$, it suffices to show that $Q_{8} \subseteq G L_{4}(2)$. It is then enough to prove that $Q_{8} \subseteq A_{8}$ on account of the exceptional isomorphism $G L_{4}(2) \cong A_{8}$. (For a proof of this well-known fact, see e.g., [2], P. 277.) Consider the left regular representation of $Q_{8}$. It provides an embedding $Q_{8} \subseteq S_{8}$. In fact, each element of order 4 in $Q_{8}$ is represented as a product of a pair of cycles of length 4 , and such a permutation is even, hence lies in $A_{8}$. Since $Q_{8}$ is generated by its elements of order 4 we obtain $Q_{8} \subseteq A_{8}$.

Claim 4. Let $1 \neq z \in Z(Q)$, and set $H_{0}:=[H, z]$. Then $\left|H_{0}\right|=2$.

This is the same as the assertion $\left|C_{H}(z)\right|=8$. To see this, let $P \subseteq G L_{4}(2)$ be the stabilizer of some hyperplane of $H$, call it $H_{1}$. Then $P \cong D \rtimes L$ with $D \cong \mathbf{Z}_{2}^{3}, L \cong G L_{3}(2)$, moreover $D$ acts trivially on $H_{1}$. Notice that $P$ contains a Sylow 2-subgroup of $G L_{4}(2)$, so that by Sylow's theorem we may assume that $Q \subseteq P$. If $z \in D$ then our assertion follows, whereas if $z \notin D$ then $Q \cap D=1$, so that $Q$ is isomorphic to a Sylow 2-subgroup of $P / D \cong L$. But this is impossible because a Sylow 2-subgroup of $G L_{3}(2)$ is dihedral of order 8 .

Claim 5. $\lambda$ exists.

We assert that

$$
H_{0}=\bigcap_{1 \neq x \in Q}[H, x] .
$$

It suffices to show that $H_{0} \subseteq[H, x]$ whenever $x \in Q$ has order 4 . To see this, suppose that $x$ has order 4 . Then $x^{2}=z$. We have

$$
\begin{aligned}
{\left[h, x^{2}\right] } & =h^{-1} x^{-2} h x^{2}=\left(h^{-1} x^{-1} h x\right)\left(x^{-1} h^{-1} x^{-1} h x^{2}\right) \\
& =[h, x][h, x]^{x}=[[h, x], x] \in[H, x],
\end{aligned}
$$

as needed.

To get the existence of $\lambda$, choose any hyperplane $H_{2}$ of $H$ that does not contain $H_{0} . H_{2}$ exists thanks to Claim 4 . Choose $\lambda \in \hat{H}$ so that $H_{2}:=k e r \lambda$. Since $H_{0} \subseteq[H, x]$ for all $x \in G \backslash H$ by (2) then $[H, x] \nsubseteq \notin k e r \lambda$.

Claim 6. $\nu_{2}(\chi)=+1$.

We have $\nu_{2}(\chi)=|G|^{-1} \sum_{g \in G} \chi\left(g^{2}\right)$. Because $\chi$ is induced from the normal subgroup $H$ then $\chi$ vanishes on $G \backslash H$. Moreover, $g^{2} \in H$ if, and only if, 
$g \in H\langle z\rangle$. If $g \in H$ then $\chi\left(g^{2}\right)=\chi(1)=8$. If $g \in H z$, say $g=h z$, then $g^{2}=[h, z] \in H_{0}$. Moreover, there are just 8 choices of $h$ with $[h, z]=1$, and for these we again have $\chi\left(g^{2}\right)=8$. As for the 8 choices satisfying $g^{2}=[h, z] \neq 1$, by construction we have $\lambda\left(g^{2}\right)=-1$, moreover $g^{2} \in Z(G)$. Therefore

$$
\chi\left(g^{2}\right)=|H|^{-1} \sum_{t \in G} \lambda\left(t^{-1} g^{2} t\right)=|H|^{-1}|G| \lambda\left(g^{2}\right)=-8 .
$$

The Claim follows from these calculations.

\section{References}

1. P. Bantay, Frobenius-Schur indicators, the Klein bottle amplitude, and the principle of orbifold covariance, Phys. Lett. B, No. 488 (2000), 207-2010.

2. J. Conway and N. Sloane, Sphere Packings, Lattices and Groups, Grundlehren Vol. 290, Springer, New York, 1988.

3. C. Curtis and I. Reiner, Methods of Representation Theory with Applications to Finite Groups and Orders, Vol. 1, Wiley-Interscience, New York, 1981.

4. J. Fuchs, I. Runkel and C. Schweigert, A reason for fusion rules to be even, J. Phys A, 35 (2002), 255-259.

5. Z. Wang, Topological Quantum Computation, CBMS Regional Conference Series in Mathematics, Vol. 112, Amer. Math. Soc., 2010. 